\title{
STRUCTURE AND EVOLUTION OF GLANT MOLECULAR CLOUDS
}

\author{
Antony A. Stark \\ AT\&T Bell Laboratories \\ HOH L231, Box 400 \\ Holmdel, NJ 07733 \\ USA
}

The mechanism of spiral structure in galaxies is a puzzle that is only partly understood. Galaxies do not revolve like solid bodies, so the spiral patterns cannot be entirely material in nature. Yet the observable tracers of spiral structure are unquestionably material: young stars, dust and gas. These objects must be organized by a collective, wave phenomenon, the spiral density wave in the disk star population. The density wave is a small $(\approx 10 \%)$ local increase in the density of stars. The interstellar medium responds to the increased gravitational force by forming Giant Molecular Clouds (GMCs), concentrations of $10^{5} \mathrm{M}_{\odot}$ or more of interstellar matter in a region about $50 \mathrm{pc}$ across. In a sense the GMCs are the spiral arms: in other galaxies, dust, gas and young star populations trace spiral structure; in the solar vicinity, these populations are seen to be associated with GMCs. This paper briefly reviews observational data supporting the hypothesis that spiral structure results from the agglomerative build-up of GMCs from smaller clouds, that this growth occurs preferentially in spiral arms, and that GMCs subsequently self-destruct because of the formation of massive stars.

In the molecular ring $5 \mathrm{kpc}$ from the galactic center, about $1 / 4$ of all interstellar material is found in GMCs (Solomon and Sanders 1980): GMCs of $10^{5}$ to $10^{6} \mathrm{M}_{\odot}$ dominate the interstellar medium, an indication that they grow efficiently at the expense of the rest of the interstellar medium. Scoville and Hersh (1979) interpreted this as meaning that GMCs are long-lived; however, this possibility is ruled out by recent galactic CO surveys (Cohen et al. 1980; Stark 1979, 1983, 1986; Solomon 1986), which find GMCs only in spiral arms, implying that GMCs are transitory objects. GMCs not only grow efficiently, they must also grow surprisingly quickly - on timescales of order $10^{7}$ years.

The data in a galactic CO survey is antenna temperature, which is a scalar function of three dimensions: galactic coordinates, $l$ and $b$, and radial velocity, $v$. GMCs can be recognized and located in this space. A GMC is $\mathrm{a} \approx 50 \mathrm{pc}$ region, emitting a peak $\mathrm{CO}$ line antenna temperature in excess of a few degrees $K$, where the line velocity does not vary more than a few $\mathrm{km} \mathrm{s}^{-1}$. It is possible to make a complete catalog of all GMCs in some extensively observed regions of the galaxy. When this is done, it is found that there exist large voids, containing few or no GMCs (Stark 1979, 1983; Cohen et al. 1980). These voids are at velocities that are permitted by galactic rotation, so that they would be populated by GMCs if the GMCs were uniformly distributed in space and rotating in circular orbits; they should contain numbers of GMCs comparable to adjacent regions of similar size, but they do not. Low mass molecular clouds are present throughout the voids and the adjacent regions. Figure 1 shows the presence of the voids in the Bell Laboratories CO Survey (Stark 1983, 1986). These results have recently been confirmed by the large-scale 

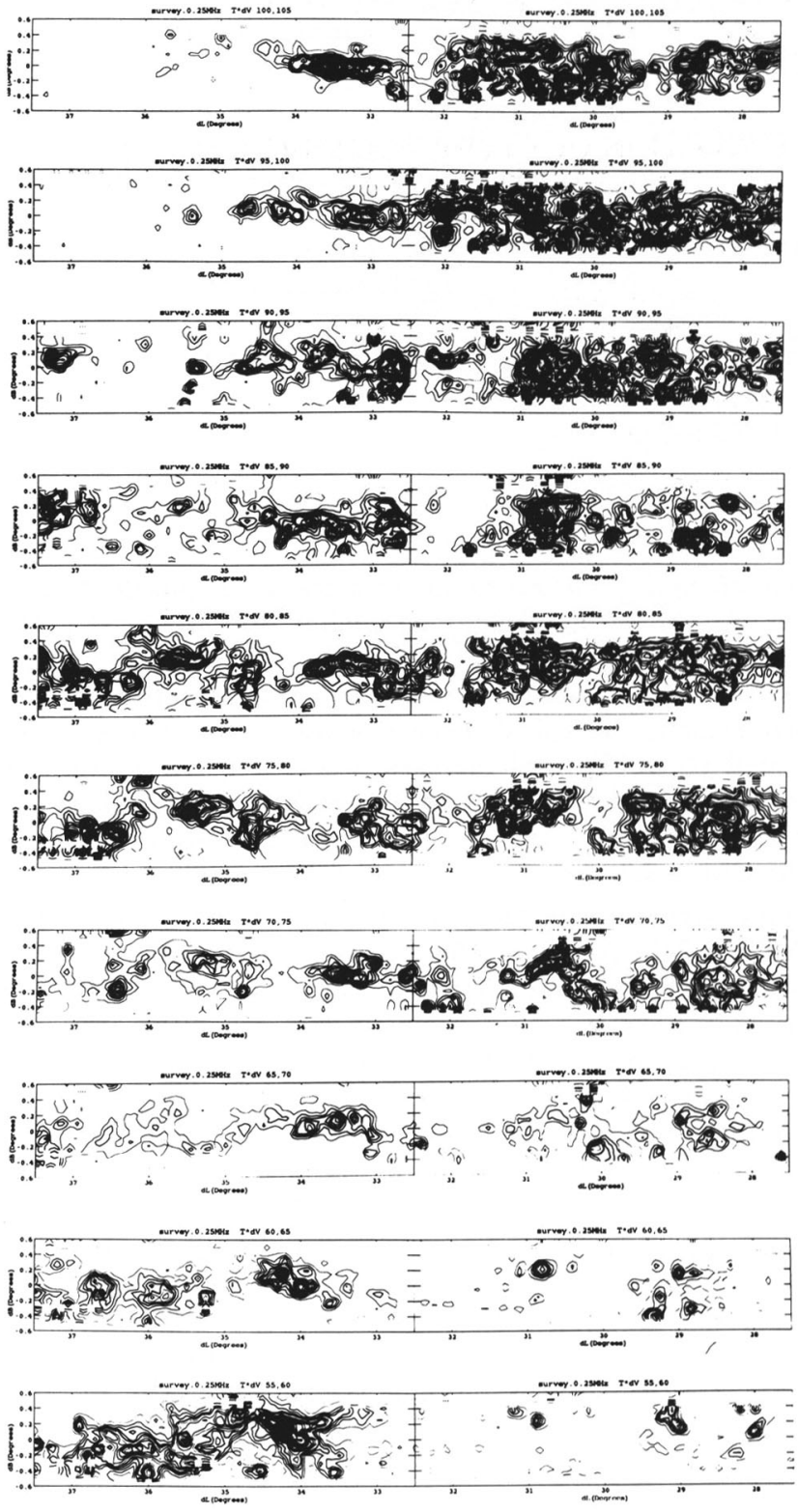

Figure 1. Contour maps of ${ }^{13} \mathrm{CO}$ integrated brightness from the Bell Laboratories CO Survey (Stark 1983). Each map covers $l=27^{\circ} .5$ to $37^{\circ} .5, b=-0^{\circ} .5$ to 0.5 . The map at top is centered at $v=102.5 \mathrm{~km} \mathrm{~s}^{-1}$, and each successive map is at $5 \mathrm{~km} \mathrm{~s}^{-1}$ lower velocity. Contour levels are spaced at $0.5 \mathrm{~K} \cdot \mathrm{km} \mathrm{s}^{-1}$ intervals. Note the absence of GMCs at $l=30^{\circ}$, $v=65 \mathrm{~km} \mathrm{~s}^{-1}$ and $l=36^{\circ}, v=90 \mathrm{~km} \mathrm{~s}^{-1}$. 
Stonybook - University of Massachusetts CO survey (Solomon 1986), were catalogs were made of cloud centers, sorted by the peak temperature at the center. GMCs, which have high brightness temperature were found only over a restricted region of $l, b, v$ space, whereas low brightness dark clouds were found to have a more uniform distribution.

The voids exist because GMCs are concentrated in the spiral arms, and they are on non-circular orbits. The contrast between the voids and their surroundings is too great to allow purely kinematic explanations. CO surveys differ in this respect from $\mathrm{HI}$ surveys. Burton (1970) has pointed out that the $l, b, v$ structure of HI surveys can be explained by a uniform spatial distribution of gas with appropriate choice of streaming velocities; however, the the arm-interarm contrast in HI much less than that of the GMCs, so this possible explanation does not apply to GMCs. The voids in the GMCs must be several square kiloparsecs in size, looking down on the disk of the galaxy, and mapping velocity into distance by the galactic rotation law. Since GMCs are not present in interarm regions, their lifetimes must be less than the time it takes gas to cross an arm, about 100 million years.

If GMCs build up in less than this time, say 30 million years, the only plausible source of material is the small molecular clouds found throughout the interarm regions. Indeed, some GMCs appear to be loose associations of smaller clouds. Figure 2 is a map of the GMC associated with the Rosette Nebula (Blitz and Stark 1986). There is structure on all

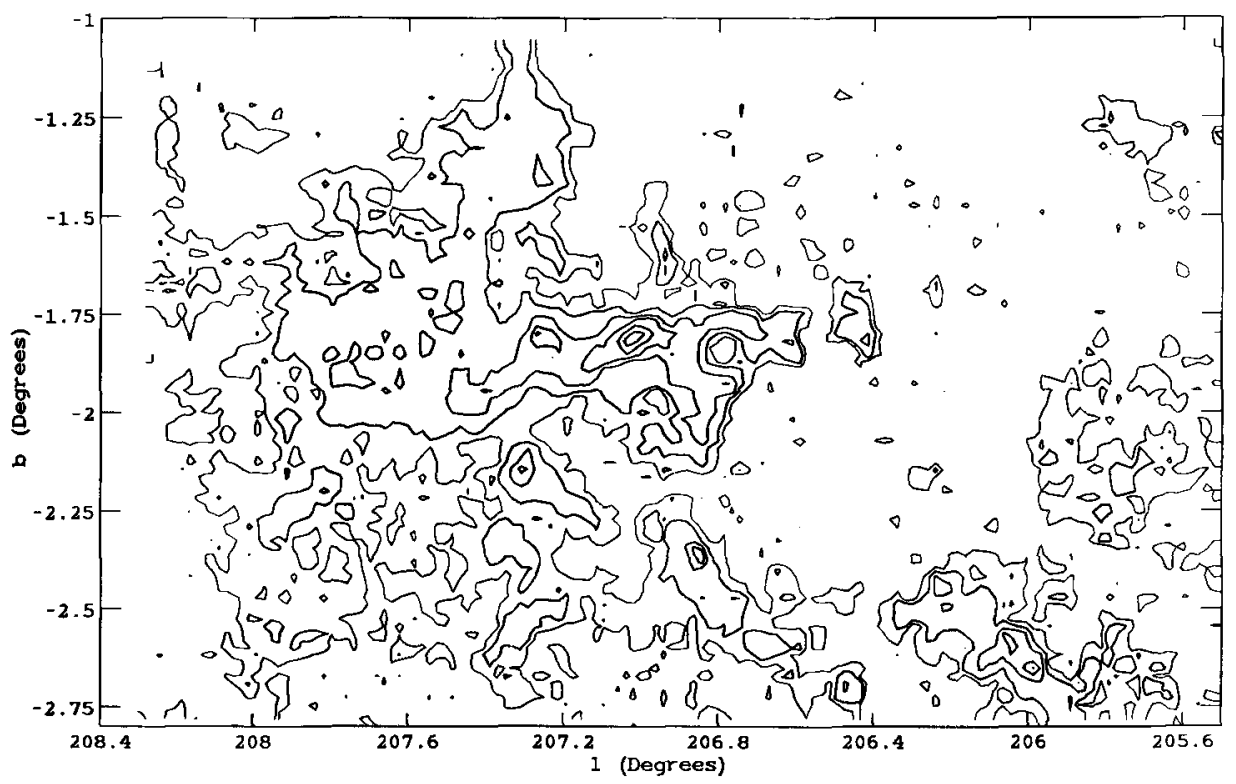

Figure 2. Contour map of integrated $\mathrm{CO}$ brightness in the Rosette Molecular Cloud. Contours are at $10,20,40,60,80$ and $100 \mathrm{~K} \cdot \mathrm{km} \mathrm{s}^{-1}$. Ordinate and abscissa are galactic coordinates. 
scales, and the cloud does not have the appearance of a smooth, centrally-condensed core. Instead, it is clumpy and full of holes, as in the model of Zuckerman and Evans (1974). The cloud is made of several hundred clumps, with masses ranging from 10 to $1000 \mathrm{M}_{\odot}$. The volume filling factor of the clumps is small. The clumps have volume densities $n \approx 10^{2}$ to $10^{5} \mathrm{~cm}^{-3}$. Their relative velocities inside the GMC are such that they are bound together. This GMC appears as if it were made of hundreds of small clouds that have lost some of their random kinetic energy, and have become bound by mutual gravitational attraction.

There is kinematic evidence that the GMCs seen in galactic CO surveys were formed by agglomeration of small clouds. Most interstellar clouds have peculiar velocities (with respect to purely circular orbits in the galaxy) of about $8 \mathrm{~km} \mathrm{~s}^{-1}$. This is true for Hi clouds of a few solar masses, and it is true for molecular clouds having $10^{4} \mathrm{M}_{\odot}$. However, it is not true for GMCs; GMCs as a group have a velocity dispersion of only a few $\mathrm{km} \mathrm{s}^{-1}$ (Stark 1979, 1983; Clemens 1985). A natural explanation is that the small molecular clouds collide to form GMCs and dissipate random kinetic energy, so that the random velocity of the combined cloud is less.

Agglomerative formation of GMCs also gives a natural explanation for the heavyended nature of the molecular cloud mass distribution (Kwan 1979; Cowie 1980, 1981): all the mass is in large clouds, because only the large clouds can grow efficiently, and they grow at the expense of the small clouds. If a cloud has a mass greater than about $2 \times 10^{5} \mathrm{M}_{\odot}$, then it will significantly perturb the orbits of nearby molecular clouds by its gravitational attraction, so that they collide and dissipate energy, and fall in to the large cloud. The cross-section of the GMC may be increased by magnetic field effects. For clouds more massive than some critical mass, the agglomeration process will be very efficient. A GMC might double its mass in a single orbit of the galaxy (Toomre 1985). Unless something prevents further growth, agglomeration runs away, and the cloud will rapidly consume the entire interstellar medium. The intervening process is the selfdestruction of GMCs through star-formation.

Between the clumps in the Rosette Molecular Cloud is relatively low density gas $\left(\langle n\rangle \approx 2 \mathrm{~cm}^{-3}\right.$ ) which is not bound to the cloud. The rate at which this gas escapes sets an upper limit to the cloud lifetime of about $10^{8} \mathrm{yr}$. Molecular gas has been removed from other parts of the cloud. The roughly circular hole at $l=206^{\circ}: 3, b=-2^{\circ} .2$ with diameter $\approx 0.7^{\circ}$ is the position of the Rosette Nebula Hil region. This hole formed as the result of destruction and removal of molecular material by the bright young stars in the Rosette. The stars themselves were formed from the cloud - when a GMC becomes sufficiently massive, it forms luminous stars, thereby indirectly destroying itself. This phenomenon has been seen in many other GMCs.

Giant Molecular Clouds play an important role in spiral structure. The external manifestations of spiral structure in galaxies, namely dust lanes, young stars, and gas, are all comprised by GMCs. GMCs are strongly concentrated to spiral arms in other galaxies. In the Milky Way, it is impossible to be sure where the spiral arms are, because of our peculiar perspective, but GMCs are certainly concentrated in some regions, and absent in others. GMCs are lumpy, as if they were concreted from smaller clouds. GMCs have a lower velocity dispersion that small clouds, also consistent with an agglomerative formation. Finally, GMCs can be seen to evaporate, and molecular gas in GMCs is destroyed by HII regions.

\section{References}

Blitz, L. and Stark, A. A. 1986 Ap. J. (Letters), 300.

Burton, W. B. 1970 Astr. Ap., 10, 76.

Clemens, D. 1985 Ap. J., 295, 422. 
Cohen, R. S., Cong, H., Dame, T. M. and Thaddeus, P. 1980 Ap. J. (Letters), 239, L53.

Cowie, L. L. 1980 Ap. J., 238, 862.

Cowie, L. L. 1981 Ap. J., 245, 66.

Kwan, J. $1979 A p . J ., \mathbf{2 2 9}, 567$.

Scoville, N. Z. and Hersh, K. 1979 Ap. J., 229, 578.

Solomon, P. M. and Sanders, D. B. 1980 in Giant Molecular Clouds in the Galaxy, ed. P. M.

Solomon and M. G. Edmunds (Pergamon: Oxford) p. 41.

Solomon, P. M. 1986 in Proc. I.A.U. 115 in press.

Stark, A. A. 1979 Ph. D. thesis, Princeton University.

Stark, A. A. 1983 in Kinematics, Dynamics and Structure of the Milky Way ed. W. L. H. Shuter (Reidel: Dordrecht) p. 127.

Stark, A. A. 1986 in Proc. I.A.U. 115 in press.

Toomre, A. 1985 personal communication.

Zuckerman, B. and Evans 1974 Ap. J. (Letters), 192, L149. 